Check for updates

Cite this: RSC Adv., 2018, 8, 15933

Received 27th December 2017 Accepted 23rd April 2018

DOI: $10.1039 / c 7 r a 13689 g$

rsc.li/rsc-advances

\section{Manufacturing of open-cell aluminum foams via infiltration casting in super-gravity fields and mechanical properties $\uparrow$}

\begin{abstract}
Zhe Wang, (D) * Jintao Gao, Kuan Chang, Long Meng, Ning Zhang and Zhancheng Guo
Replicated open-cell aluminum foams were produced by infiltration casting in super-gravity fields. Infiltration of preforms packed by $\mathrm{NaCl}$ particles with different sizes was conducted to demonstrate the technical feasibility of this method. The relative densities between 0.25 and 0.34 of the aluminum foams were obtained by varying the $\mathrm{NaCl}$ particle size of the preform from 600 to $200 \mu \mathrm{m}$. Increasing the gravity coefficient $(G)$ increased the centrifugal pressure $\left(P_{c}\right)$ and correspondingly improved the relative densities and structural integrity of the resulting foams. As $P_{\mathrm{c}}$ increased, the aluminum foam exhibited a transition from a structure of smooth struts to a relatively complex structure where many protrusions extended inside the pores from the surface of the struts. Also, the specific relationship between the minimum centrifugal pressures necessary to produce self-standing aluminum foams and the $\mathrm{NaCl}$ particle size of the preform was established. The minimum centrifugal pressures of 32,49 and $83 \mathrm{kPa}$ were required for aluminum foams with pore sizes of 600, 400 and $200 \mu \mathrm{m}$, respectively. Preliminary results show that super-gravity infiltration is promising to be a practical manufacture process for replicated open-cell aluminum foams.
\end{abstract}

\section{Introduction}

Open-cell metallic foams have been manufactured using a number of different methods such as infiltration casting, powder metallurgy ${ }^{1}$ and electro-deposition. ${ }^{2}$ Among these, the infiltration casting offers the possibility to highly control foam topology (shape, size and distribution of pores). This method usually uses a removable preform with interconnected porous structure, into which a molten metal infiltrates under external pressure, before removal of the preform to leave an open-cell structure. ${ }^{3}$

Since infiltration casting was first proposed to produce metallic foams in 1961 by Polonsky et al., ${ }^{\mathbf{4}}$ many various preform designs and devices have been developed. A detailed review on the infiltration casting for production of metallic foams can be found elsewhere. ${ }^{5}$ Fabrizio et al. ${ }^{6}$ used hydraulic cylinder to provide the pressure of $2.5 \mathrm{MPa}$ and produced aluminum foams by replication of a $\mathrm{NaCl}$ preform. LaraRodriguez et $a .^{7}$ developed a replication casting device and successfully fabricated open-cell magnesium foams with pore size of $3.3 \mathrm{~mm}$ under an argon gas pressure of 1.96 bar. The conventional infiltration casting method mainly utilizes mechanical pressure (piston or gas), however, it is insufficient

State Key Laboratory of Advanced Metallurgy, University of Science and Technology Beijing, Beijing 100083, China. E-mail: zhewang@ustb.edu.cn

$\dagger$ Electronic supplementary information (ESI) available. See DOI: $10.1039 / \mathrm{c} 7 \mathrm{ra13689g}$ to effectively overcome the surface tension of liquid metals and the residue gas in the interstices or voids of preform is hard to be completely squeezed out, hence, it is difficult to achieve the complete fill of the preform with molten metals. ${ }^{8-10}$

It is known that under super-gravity fields (created by a centrifugal apparatus), the mass transfer and migration of heterogeneous phases can be enhanced immensely. ${ }^{11}$ Driven by these qualities, the super-gravity technology has been successfully applied in many fields so far, such as chemical industry (distillation, $^{12}$ absorption ${ }^{13}$ and sulfonation ${ }^{14}$ ), metallurgy (purification of metals ${ }^{\mathbf{1 5}}$ and valuable element recovery from slag melts ${ }^{\mathbf{1 6}}$ ) and material preparation. In terms of material preparation, super-gravity infiltration, also known as centrifugal casting, is one of the simplest and cost effective technologies for producing metallic complex pieces, functionally graded materials and metal matrix composites (MMCs). Huang et al. ${ }^{17}$ fabricated $\mathrm{Al}-\mathrm{Si}$ alloy-based pistons reinforced with $\mathrm{SiC}$ by vertical centrifugal casting and reported that the SiC and primary Si particles were mainly distributed towards the piston head, correspondingly increasing the wear resistance and hardness. Wannasin and Flemings ${ }^{\mathbf{1 8}}$ developed a super-gravity infiltration apparatus which can achieve pressures up to $15 \mathrm{MPa}$ and successfully fabricated MMCs reinforced with $300 \mathrm{~nm}$ ceramic powders.

Super-gravity infiltration can also be a feasible process to fabricate open-cell metallic foams. Under super-gravity fields, the negative effect on infiltration due to the surface tension of molten metal can be reduced even eliminated, ${ }^{\mathbf{1 1} 19}$ easily 
resulting in a complete formation of the open-cell foam. The study on manufacturing metallic foam via super-gravity infiltration is quite limited to the best of our knowledge. SánchezMartínez et $a l .^{20}$ firstly obtained open-cell zinc alloy foams using prepared $\mathrm{NaCl}$ preforms by centrifugal casting and experimentally determined the minimum centrifugal forces required to fully form the zinc foams. Yet, the effects of centrifugal force on relative density and structure of resulted foams were not reported. Also, it is noted that the minimum centrifugal force is determined by several parameters such as $\mathrm{NaCl}$ particle size and infiltration temperature. It is difficult to isolate the effect of a specific parameter on minimum centrifugal force using conventional centrifugal casting where the infiltration temperature cannot be accurately controlled.

In our previous study, the 3-D open-cell aluminum foams were successfully prepared using plaster-foam preforms by infiltration casting under super-gravity fields. ${ }^{19}$ It is found that increasing the gravity coefficient could effectively overcome the resistance of molten aluminum infiltrating into the channels of preform, thus significantly enhancing the quality of the final aluminum foams. However, after solidification, the removal of plaster materials from the plaster-aluminum composite often resulted in the damage of foam structure, so neither the infiltration parameters nor the mechanical properties of aluminum foams were obtained unfortunately.

In comparison to plaster-foam preform, $\mathrm{NaCl}$ preform can be easily removed by water leaching and is a more common and practical choice to produce open-cell aluminum foams. Therefore, in this study, the preforms constituted by packed $\mathrm{NaCl}$ particles were used to further explore the feasibility of the super-gravity infiltration in fabricating replicated opencell aluminum foams. Using the self-developed experimental super-gravity device with the accurate control of infiltration temperature, the specific relationship between the minimum centrifugal pressures as a function of the particle size of preform was established. The effects of the super-gravity field on the structure, relative density and mechanical properties of the prepared aluminum foams were also investigated.

\section{Experimental}

\subsection{Materials}

A 99.99\% pure aluminum (Southwest Aluminum Ltd., China) was used in this study. The commercial $\mathrm{NaCl}$ particles $(>99 \%$ pure, supplied by Sinopharm Chemical Reagent Ltd., China) with cubic morphology were used to prepare preforms. The original $\mathrm{NaCl}$ particles were dried in an oven at $110{ }^{\circ} \mathrm{C}$ for 4 hours before being screened to obtain four particle size ranges of 150-250 $\mu \mathrm{m}, 300-500 \mu \mathrm{m}$ and 500-700 $\mu \mathrm{m}$. The NaCl particle sizes were classified here as (i) $200 \mu \mathrm{m}$, (ii) $400 \mu \mathrm{m}$ and (iii) 600 $\mu \mathrm{m}$, considering each average size. The majority of $\mathrm{NaCl}$ particles used for the preform presented a cubic shape, while only a few particles had a relatively smooth outline, which was particularly obvious in the particles of $600 \mu \mathrm{m}$ (Fig. S1, ESI†).

\subsection{Infiltration experiments}

The super-gravity fields used in the infiltration experiments were generated by a centrifugal apparatus. The image and schematic of the centrifugal apparatus are presented in Fig. 1. The setup utilizes a resistance heating furnace with a cylindrical alumina chamber of $40 \mathrm{~mm}$ diameter and $150 \mathrm{~mm}$ length, and an R-type thermocouple was used to monitor and control the temperature. The heating furnace and a counterweight were fixed onto the centrifugal rotor symmetrically. The dashed line shown in Fig. 1(b) indicates the furnace and counterweight in a stationary status and they changed from vertical to horizontal and rotated therewith once the centrifugal rotor started running.

In order to stop the NaCl preform floating toward the center of rotation in the super-gravity field due to the lower density of $\mathrm{NaCl}$ $\left(2.165 \mathrm{~g} \mathrm{~cm}^{-3}\right.$ at $\left.20{ }^{\circ} \mathrm{C}\right)$ than aluminum $\left(2.7 \mathrm{~g} \mathrm{~cm}^{-3}\right.$ at $\left.20{ }^{\circ} \mathrm{C}\right)$, a graphite crucible consisting of two small ones was used in the infiltration experiment (Fig. S2, ESI $\dagger$ ). The two small crucibles

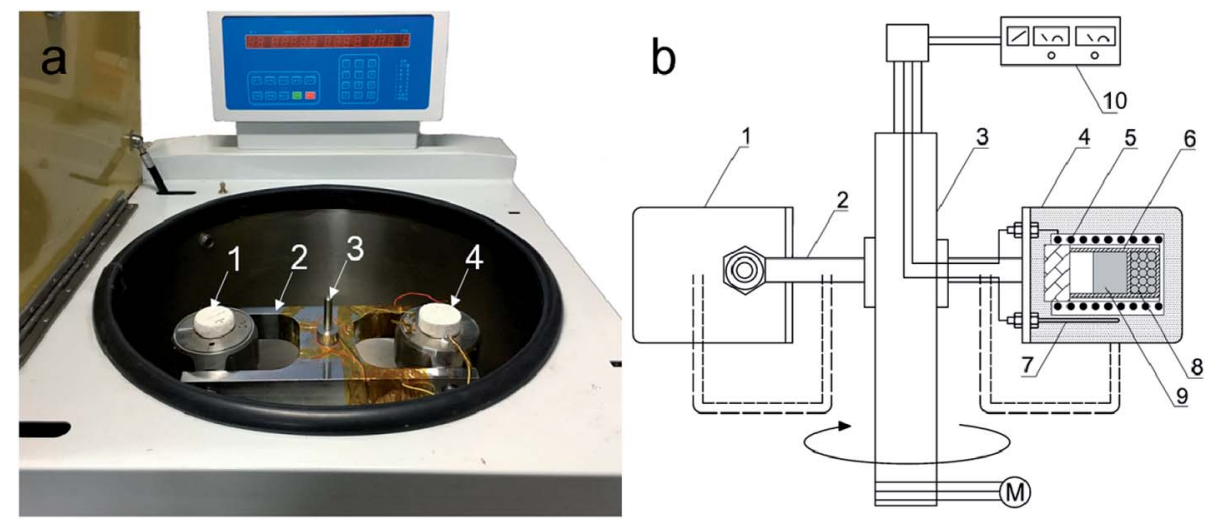

Fig. 1 Image (a) and schematic (b) of the centrifugal apparatus: (1) counterweight, (2) centrifugal rotor, (3) centrifugal axis, (4) resistance heating furnace, (5) resistance coil, (6) graphite crucible, (7) type-R thermocouple, (8) NaCl preform, (9) aluminum melt, (10) temperature controller. 
had the same internal diameter of $21 \mathrm{~mm}$ and there were 20 filtering holes with a diameter of $0.5 \mathrm{~mm}$ in the bottom of the upper crucible. To simplify the fabrication procedure, preform sintering was avoided and the $\mathrm{NaCl}$ particles were directly placed into the lower graphite crucible as the preform; while about $30 \mathrm{~g}$ aluminum blocks were placed into the upper graphite crucible. Then the upper one was screwed tightly onto the lower one, constituting a whole crucible. This whole crucible was then heated at $710{ }^{\circ} \mathrm{C}$ for $20 \mathrm{~min}$ in the heating furnace of the centrifugal apparatus (Fig. 1) to make aluminum blocks completely melted. Later, the apparatus was started and adjusted to the desired rotational speed. Driven by the centrifugal force, the molten aluminum went through the filtering holes and infiltrated into the voids of the $\mathrm{NaCl}$ preform. The apparatus was kept rotating at $710{ }^{\circ} \mathrm{C}$ for $10 \mathrm{~min}$ and later the temperature decreased at a cooling rate of $15^{\circ} \mathrm{C} \mathrm{min}^{-1}$. When the temperature was below $500{ }^{\circ} \mathrm{C}$ (the aluminum was fully solidified), the centrifugal apparatus was shut off and the graphite crucible with sample was taken out and cooled in air. The formed aluminum$\mathrm{NaCl}(\mathrm{Al} / \mathrm{NaCl})$ composite was took out from the lower crucible and machined to the desired size for analysis. Finally, the $\mathrm{NaCl}$ was completely removed by dissolving in water from the composite to obtain the final sample.

To quantify the super-gravity field, gravity coefficient $(G)$ is defined as the ratio of centrifugal acceleration to the normal gravity acceleration, as shown in eqn (1)

$$
G=\frac{\sqrt{g^{2}+\left(\omega^{2} r\right)^{2}}}{g}=\frac{\sqrt{g^{2}+\left(\frac{N^{2} \pi^{2} r}{900}\right)^{2}}}{g}
$$

where $g$ is the normal gravitational acceleration, $9.8 \mathrm{~m} \mathrm{~s}^{-2} ; \omega$ denotes the angular velocity, $\operatorname{rad~s}^{-1} ; r$ is the distance from the centrifugal axis to the center of $\mathrm{NaCl}$ preform $(0.25 \mathrm{~m}$ in this work); $N$ denotes the rotational speed, rpm. During the supergravity infiltration process, the rotation provides sufficient centrifugal pressure to obtain a full infiltration. The centrifugal pressure acting on the surface of preform $\left(P_{\mathrm{c}}\right)$ caused by centrifugal force can be calculated by

$$
P_{\mathrm{c}}=\frac{\rho \omega^{2}\left(L_{2}^{2}-L_{1}^{2}\right)}{2}=\frac{\rho N^{2} \pi^{2}\left(L_{2}^{2}-L_{1}^{2}\right)}{1800}
$$

where $\rho$ denotes the density of the molten aluminum, and $L_{2}$ and $L_{1}$ the molten aluminum levels measured from the center of rotation (Fig. S3, ESI $\dagger$ ). More detailed description can be found elsewhere. ${ }^{18}$ In this study, with the density of aluminum at $710{ }^{\circ} \mathrm{C}\left(2.373 \mathrm{~g} \mathrm{~cm}^{-3}\right), L_{2}(0.24 \mathrm{~m})$ and $L_{1}(0.206 \mathrm{~m})$, and combining with eqn (1), the centrifugal pressure is also expressed in eqn (3).

$$
P_{\mathrm{c}}=705 G
$$

Based on eqn (1)-(3), with increasing the rotational speed from $0 \mathrm{rpm}$ to $1891 \mathrm{rpm}$ (maximum in this study), the generated gravity coefficient and centrifugal pressure increased from 1 to 1000 and $0 \mathrm{kPa}$ to $705 \mathrm{kPa}$, respectively.

\subsection{Characterization}

The structure of the $\mathrm{NaCl}$ particles used for preform and fabricated aluminum foam samples were examined by scanning electron microscopy (SEM, MLA 250, FEI Quanta, USA) analysis.

Cylindrical aluminum foam samples with a diameter of $20 \mathrm{~mm}$ and a height of $20 \mathrm{~mm}$ were tested in compression using a universal testing machine at a constant cross-head speed of $0.5 \mathrm{~mm} \mathrm{~min}^{-1}$. At least three samples for each pore size were examined and the average mechanical properties (e.g. yield strength, plateau stress and elastic modulus) were reported.

The relationship between the porosity $P_{\mathrm{r}}$ (\%) of the aluminum foam and the relative density $\left(\rho_{\mathrm{f}} / \rho_{\mathrm{s}}\right)$ of the foam was presented in eqn (4).

$$
P_{\mathrm{r}}=\left(1-\frac{\rho_{\mathrm{f}}}{\rho_{\mathrm{s}}}\right) \times 100
$$

where $\rho_{\mathrm{f}}$ and $\rho_{\mathrm{s}}$ represent densities of the green foam and bulk aluminum (about $2.7 \mathrm{~g} \mathrm{~cm}^{-3}$ ), respectively. The density of the aluminum foam $\rho_{\mathrm{f}}$ was determined by measuring the mass and calculating the volume from the known physical dimensions.

\section{Results}

\subsection{Infiltration casting in super-gravity fields}

Fig. 2 shows images of the aluminum foams obtained at $P_{\mathrm{c}}=$ $353 \mathrm{kPa}(G=500)$ with different pore sizes and their metallic structures. As shown in Fig. 2(a-c), the homogeneous distribution of pores in their transverse and longitudinal directions was clearly observed. Fig. 2(d-f) indicate the SEM images taken from the cross sections of the final aluminum foams. These fabricated aluminum foams obtained interconnected open porosity with shape (mostly cubic) and size equivalent to those of the $\mathrm{NaCl}$ particles used for preforms.

During the infiltration casting in super-gravity fields, the molten aluminum was forced to penetrate the channels of the
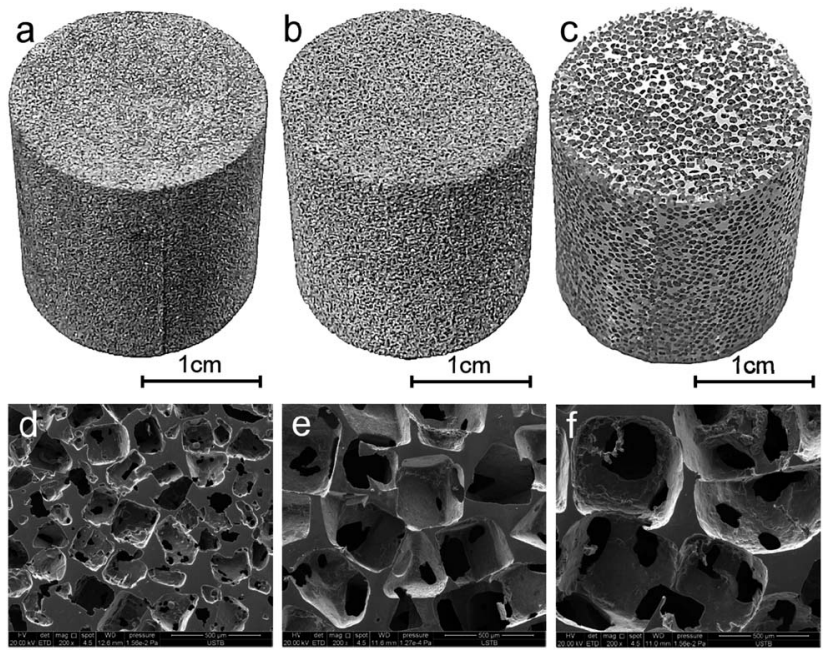

Fig. 2 Open-cell aluminum foams with different pore sizes and their interconnected structures fabricated at $P_{\mathrm{c}}=353 \mathrm{kPa}(G=500)$. (a) and (d) $200 \mu \mathrm{m}$, (b) and (e) $400 \mu \mathrm{m}$, (c) and (f) $600 \mu \mathrm{m}$. 


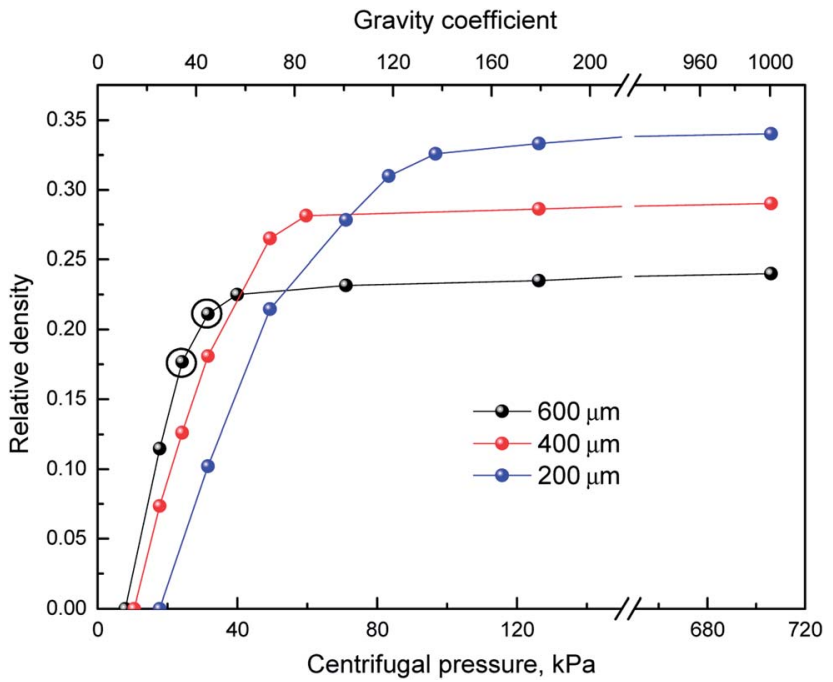

Fig. 3 Effect of centrifugal pressure and gravity coefficient on the relative densities of the final aluminum foams.

$\mathrm{NaCl}$ preforms, and three kinds of infiltration results were obtained: no infiltration, partial infiltration and full infiltration. To evaluate the influence of super-gravity field on extent of infiltration, the effect of centrifugal pressure $\left(P_{\mathrm{c}}\right)$ and gravity coefficient $(G)$ on the relative densities of the final aluminum foams were illustrated in Fig. 3. The curves were similar for all three kinds of aluminum foams, and the relative density increased gradually with increasing the centrifugal pressure and gravity coefficient. Take the $\mathrm{NaCl}$ particle size of $600 \mu \mathrm{m}$ for instance. The $\mathrm{NaCl}$ preform cannot be infiltrated by molten aluminum until $P_{\mathrm{c}}$ was higher than $8 \mathrm{kPa}(G=11)$. The relative density increased quickly to 0.21 with increasing $P_{\mathrm{c}}$ to $32 \mathrm{kPa}(G$ $=45$ ), while over $32 \mathrm{kPa}$ the relative density also increased but at a relatively slow rate. Under the maximum centrifugal pressure of $705 \mathrm{kPa}(G=1000)$, the relative density of the aluminum foam with the pore size of $600 \mu \mathrm{m}$ reach its peak at 0.24 .

In addition to relative density, the centrifugal pressure and gravity coefficient also exerted great influences on the integrity and structure of the final aluminum foams. Fig. 4 shows two typical cross-section images of the aluminum foams with a pore size of $600 \mu \mathrm{m}$ fabricated at different centrifugal pressures (24 $\mathrm{kPa}$ and $32 \mathrm{kPa}$, marked by two cycles in Fig. 3). As shown in Fig. 4(a), at $P_{\mathrm{c}}=24 \mathrm{kPa}(G=24)$, the $\mathrm{NaCl}$ preform was partially infiltrated and a number of large cavities (marked in dotted oval) were presented at the lower part of the aluminum foam. In comparison, when $P_{\mathrm{c}}$ reach $32 \mathrm{kPa}$, a self-standing aluminum foam was fabricated. It is shown from Fig. 4(b) that, at $P_{\mathrm{c}}=32$ $\mathrm{kPa}$, the $\mathrm{NaCl}$ preform was fully infiltrated and the manufactured aluminum foam was sound without any macroscopical defect. The centrifugal pressures were required to be higher than 49 and $83 \mathrm{kPa}$ to fabricate the self-standing aluminum foams with pore size of 400 and $200 \mu \mathrm{m}$, respectively. Also, it is indicated from Fig. 3 that, over 32,49 and $83 \mathrm{kPa}$, further increasing $P_{\mathrm{c}}$ improved the relative densities of foams with the pore sizes of 600,400 and $200 \mu \mathrm{m}$ at a relatively slow rate. Therefore, the pressures of 32, 49 and $83 \mathrm{kPa}$ can be regarded as

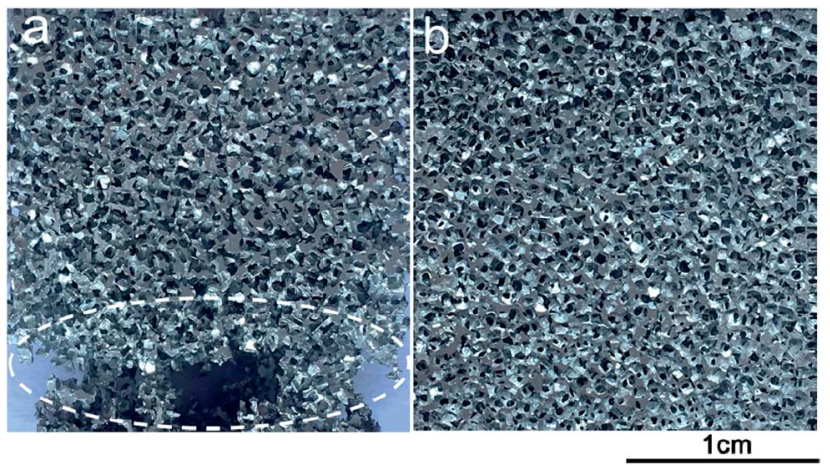

Fig. 4 Typical cross-section images of aluminum foams with a pore size of $600 \mu \mathrm{m}$ fabricated at different centrifugal pressures. (a) $24 \mathrm{kPa}$ and (b) $32 \mathrm{kPa}$.

the minimum centrifugal pressures to obtain the full infiltration of $\mathrm{NaCl}$ preforms with the $\mathrm{NaCl}$ particle sizes of 600,400 and $200 \mu \mathrm{m}$, respectively. It is indicated that to obtain a full infiltration of a preform with a smaller particle size, a larger minimum centrifugal pressure was required.

The metallic structures of aluminum foams with a pore size of $600 \mu \mathrm{m}$ obtained at different centrifugal pressures was presented in Fig. 5. Fig. 5(d-f) are the SEM images of the boxed region in Fig. $5(\mathrm{a}-\mathrm{c})$ at higher magnification, respectively. The relatively smooth struts were obtained in the aluminum foam fabricated at low $P_{\mathrm{c}}\left(32 \mathrm{kPa}\right.$, Fig. 5(a) and (d)). When $P_{\mathrm{c}}$ increased, the structure of the aluminum foams became complex and some fine aluminum protrusions (marked by arrows) were found on the main cell struts. And there was a gradual increase in the number of these aluminum protrusions as $P_{\mathrm{c}}$ increased. The formation of these protrusions increases the surface area and impedance of the foams, which can benefit their properties of filtration, catalysis and sound absorption. ${ }^{19}$

Table 1 shows the porosities and densities of the aluminum foams with different pore sizes fabricated at $P_{\mathrm{c}}=705 \mathrm{kPa}$. It is found that with increasing the particle size of $\mathrm{NaCl}$ from $200 \mu \mathrm{m}$ to $600 \mu \mathrm{m}$, the porosity of aluminum foam increased gradually from $66 \%$ to $76 \%$. The relative density of foams and density of sponges had the opposite trend with the porosity and reach the minima at the pore size of $600 \mu \mathrm{m}$ which were 0.24 and $0.65 \mathrm{~g} \mathrm{~cm}^{-3}$, respectively.

\subsection{Mechanical properties}

The typical compressive stress-strain curves of the produced aluminum foams are shown in Fig. 6 . These aluminum foams were fabricated at $P_{\mathrm{c}}=705 \mathrm{kPa}$, with the porosities and densities listed in Table 1. They exhibited the similar stress-strain behavior in comparison with the other open-cell metallic foams, which can be characterized by three distinct regions, i.e. a linear elastic region at very low strain without the peak stress, an extended collapse plateau region and a densification region where there was a sharp increase in compressive stress. Also, a relatively more horizontal plateau was obtained by the 

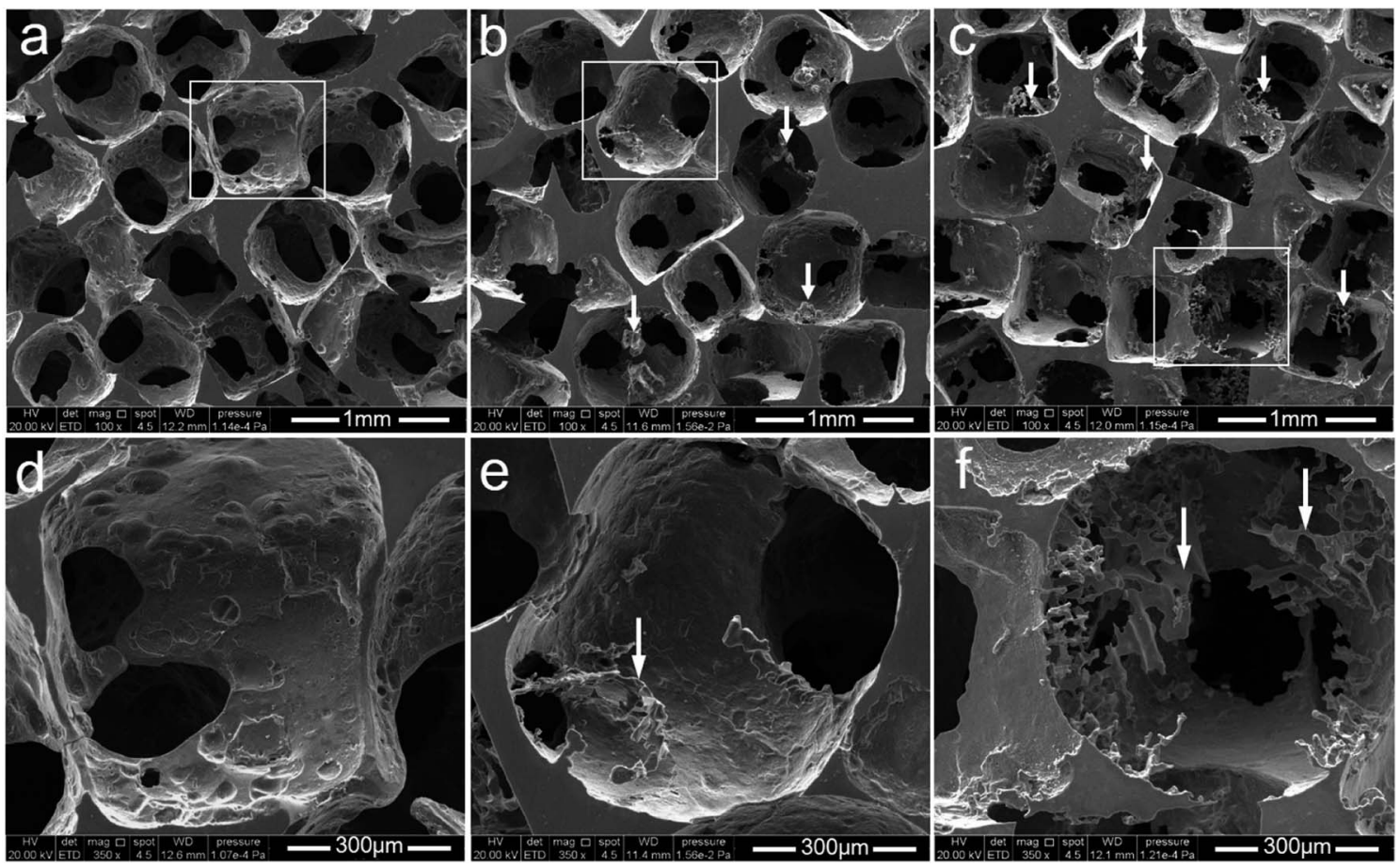

Fig. 5 SEM images of aluminum foam with a pore size of $600 \mu \mathrm{m}$ infiltrated at various centrifugal pressures. (a) and (d) $32 \mathrm{kPa}$, (b) and (e) $353 \mathrm{kPa}$, (c) and ( $f) 705 \mathrm{kPa}$. $(\mathrm{d}-\mathrm{f})$ are the SEM images of the boxed region in $(\mathrm{a}-\mathrm{c})$ respectively at higher magnification, highlighting the various morphologies of cell struts.

Table 1 Porosities and densities of the fabricated aluminum foams with different pore sizes produced at $P_{\mathrm{c}}=705 \mathrm{kPa}(G=1000)$

\begin{tabular}{llll}
$\begin{array}{l}\text { Pore } \\
\text { size, } \mu \mathrm{m}\end{array}$ & Porosity, \% & Relative density & Density of sponges, $\mathrm{g} \mathrm{cm}^{-3}$ \\
\hline 200 & 66 & 0.34 & 0.92 \\
400 & 71 & 0.29 & 0.78 \\
600 & 76 & 0.24 & 0.65 \\
\hline
\end{tabular}

aluminum foam with a lower relative density (0.24) than those with higher relative densities.

The experimental mechanical parameters of the three variant aluminum foams are summarized in Table 2 . The plateau stress $\left(\sigma_{\mathrm{pl}}\right)$ in this work was represented by the average value of the stress measured between the elastic region and the densification region. The experimental results of densification strain $\left(\varepsilon_{\mathrm{D}}\right)$ were calculated via intersection of two tangent line method and the detailed description has been portrayed elsewhere. ${ }^{21}$ It is observed from Table 2 that elastic modulus $(E)$, $0.2 \%$ yield stress $\left(\sigma_{0.2 \%}\right)$ and $\sigma_{\mathrm{pl}}$ all increased with increasing the relative density of aluminum foams. The aluminum foam with the pore size of $200 \mu \mathrm{m}$ obtained the highest relative density (0.34) and correspondingly had the relatively higher $E$ (93.3), $\sigma_{0.2 \%}(2.59 \mathrm{MPa})$ and $\sigma_{\mathrm{pl}}(5.35 \mathrm{MPa})$.

\section{Discussion}

To achieve the penetration of molten aluminum into the channel of the $\mathrm{NaCl}$ preform, the centrifugal pressure is

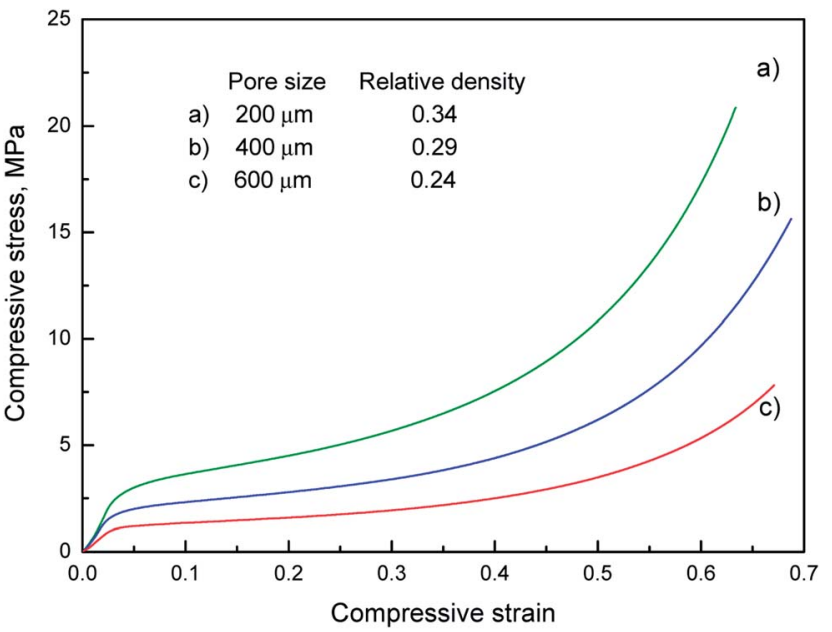

Fig. 6 Compressive stress-strain curves of aluminum foams with different pore sizes fabricated at $P_{\mathrm{c}}=705 \mathrm{kPa}(G=1000)$.

required to be larger than the flow resistance $\left(P_{\mathrm{s}}\right)$ which can be described by the capillary law:

$$
P_{\mathrm{s}}=-\frac{4 \gamma \cos \theta}{d}
$$

where $\gamma$ denotes the surface tension of molten aluminum, $\theta$ is the wetting angle and $d$ is the effective diameter of the channel of the preform. Since the flow resistance is inversely proportional to the diameter of the channel (eqn (5)), the molten aluminum only infiltrates the large channels of the $\mathrm{NaCl}$ 
Table 2 Mechanical properties of the open-cell aluminum foams

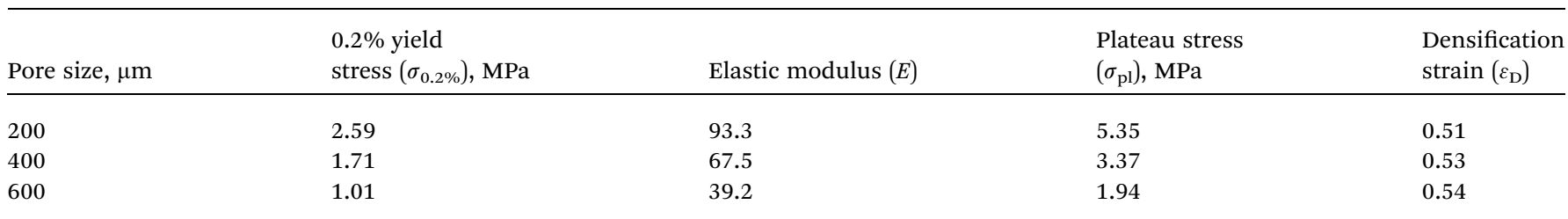

preform under low centrifugal pressures. With increasing the centrifugal pressure, the molten aluminum penetrates the narrower spaces in the preform (Fig. S4, ESI $\dagger$ ), leading to a corresponding increase in the relative density and structural integrity of the final aluminum foam (Fig. 3 and 4). It is well known that there are many deep pits existing in the $\mathrm{NaCl}$ particles, ${ }^{22,23}$ and more deep pits were infiltrated by molten aluminum at higher centrifugal pressures. Therefore, with increasing centrifugal pressures, the aluminum foams showed a gradual formation of protrusions extending inside the pores from the surface of the struts, as shown in Fig. 5.

As mentioned above, a larger minimum centrifugal pressure was required to obtain a full infiltration of a preform with a smaller particle size. It can be explained by eqn (6) which is introduced by Garcia-Cordovilla et al. ${ }^{24}$ based on capillary law for calculation of the minimum infiltration pressure $\left(P_{\mathrm{th}}\right)$ necessary for infiltration between the metal and the ceramic reinforcement:

$$
P_{\mathrm{th}}=-6 \lambda \gamma \cos \theta \frac{V_{\mathrm{p}}}{\left(1-V_{\mathrm{p}}\right) D}
$$

where $\lambda$ denotes the correcting factor for spherical deviation and surface conditions, $\gamma$ the surface tension of molten aluminum, $V_{\mathrm{p}}$ the volume fraction of the preform, $\theta$ the contact angle and $D$ the average diameter of $\mathrm{NaCl}$ particles. As shown in eqn (6), $P_{\text {th }}$ is inversely proportional to $D$, which is in good consistent with the observation in this work that reducing the $\mathrm{NaCl}$ particle size increased the minimum centrifugal pressure. It is also noted that the minimum centrifugal pressures

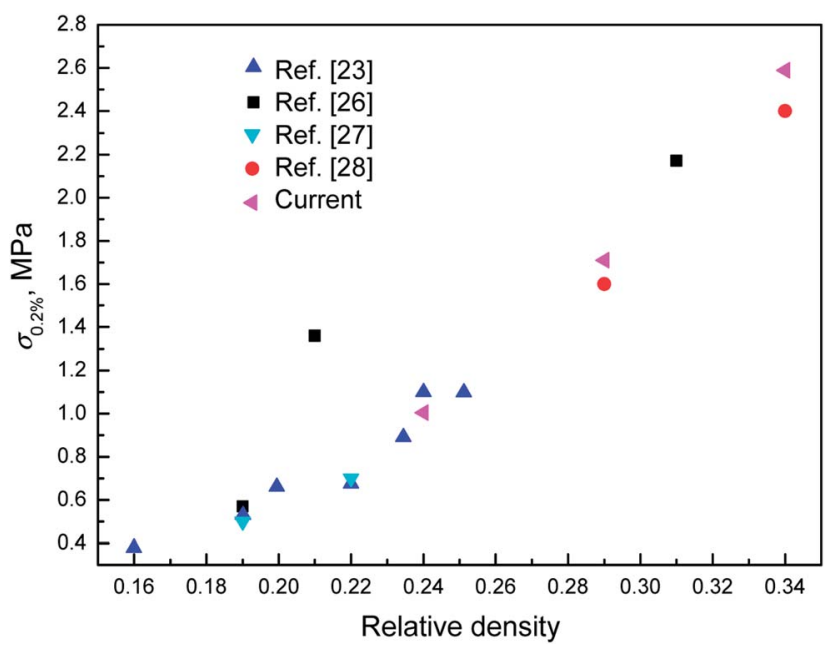

Fig. 7 The relationship between relative density and $\sigma_{0.2 \%}$ of the aluminum foams from current study and some other works. required for the fabrication of aluminum foams were quite lower than those (even higher than $10 \mathrm{MPa}$ ) used in manufacturing metal-matrix composites by the same process. ${ }^{18,25}$ It is mainly because the particle sizes of reinforcement materials in metal-matrix composites were much smaller (even nanometer level) than those of $\mathrm{NaCl}$ used for metallic foams.

Fig. 7 shows the comparison of the $\sigma_{0.2 \%}$ of the aluminum foams with different relative densities from current study and some other works ${ }^{23,26-28}$ where these aluminum foams were produced by the gas pressure or vacuum infiltration. It is observed that all aluminum foams with similar relative densities obtained the similar values of $\sigma_{0.2 \%}$. Therefore, it can be seen that the qualities of the aluminum foams produced in this work by super-gravity infiltration can compare with those fabricated by the traditional gas pressure or vacuum infiltration. Given that the centrifugal casting has been successfully applied to fabricating metal-matrix composites as a cost effective and simple method, which requires much higher centrifugal pressures ${ }^{\mathbf{1 8 , 2 5}}$ than those in manufacturing aluminum foams, the super-gravity infiltration is promising to be practically applied in fabricating replicated open-cell aluminum foams. Further study on scaling up the super-gravity infiltration device for producing metallic foams is in progress.

\section{Conclusions}

(1) Replicated open-cell aluminum foams with pore sizes of 200 , 400 , and $600 \mu \mathrm{m}$ were successfully manufactured by infiltration casting in super-gravity fields. The relative density between 0.25 and 0.34 of the aluminum foams were obtained by varying the $\mathrm{NaCl}$ particle size of the preform.

(2) Increasing gravity coefficient and centrifugal pressure greatly improved the relative densities and structural integrity of the fabricated aluminum foams. At higher centrifugal pressures, the aluminum foams showed a gradual formation of protrusions extending inside the pores from the surface of the struts.

(3) The specific relationship between the minimum centrifugal pressures necessary to produce self-standing aluminum foams and the NaCl particle size of preform was established. The minimum centrifugal pressures of 32, 49 and $83 \mathrm{kPa}$ were required for aluminum foams with pore size of 600,400 and 200 $\mu \mathrm{m}$, respectively.

(4) Preliminary results show the super-gravity infiltration is promising to be a practical method for producing replicated open-cell aluminum foams. 


\section{Conflicts of interest}

There are no conflicts to declare.

\section{Acknowledgements}

This work was supported by the National Natural Science Foundation of China (No. 51704022) and the Fundamental Research Funds for the Central Universities (FRF-TP-16-036A1) and the project of State Key Laboratory of Advanced Metallurgy (41617002).

\section{References}

1 J. Jia, A. R. Siddiq and A. R. Kennedy, Porous titanium manufactured by a novel powder tapping method using spherical salt bead space holders: characterisation and mechanical properties, J. Mech. Behav. Biomed. Mater., 2015, 48, 229-240.

2 D. Jose, V. Zanardi and S. S. Sundarram, Fabrication of bulk graded microcellular nickel foams using combined electroless and electroplating of polymer sphere template, Manuf. Lett., 2016, 8, 1-5.

3 M. Vesenjak, M. A. Sulong, L. Krstulović-Opara, M. Borovinšek, V. Mathier and T. Fiedler, Dynamic compression of aluminium foam derived from infiltration casting of salt dough, Mech. Mater., 2016, 93, 96-108.

4 L. Polonsky, S. Lipson and H. Markus, Light weight cellular metal, Mod. Cast., 1961, 39, 4.

$5 \mathrm{~J}$. Banhart, Manufacture, characterisation and application of cellular metals and metal foams, Prog. Mater. Sci., 2001, 46, 559-632.

6 Q. Fabrizio, A. Boschetto, L. Rovatti and L. Santo, Replication casting of open-cell $\mathrm{AlSi}_{7} \mathrm{Mg}_{0.3}$ foams, Mater. Lett., 2011, 65, 2558-2561.

7 G. Lara-Rodriguez, I. Figueroa, M. Suarez, O. Novelo-Peralta, I. Alfonso and R. Goodall, A replication-casting device for manufacturing open-cell $\mathrm{Mg}$ foams, J. Mater. Process. Technol., 2017, 243, 16-22.

8 D. Huo, J. Yang, X. Zhou, H. Wang and T. Zhang, Preparation of open-celled aluminum foams by counter-gravity infiltration casting, Trans. Nonferrous Met. Soc. China, 2012, 22, 85-89.

9 S. Zhou, X. Zhang, Z. Ding, C. Min, G. Xu and W. Zhu, Fabrication and tribological properties of carbon nanotubes reinforced $\mathrm{Al}$ composites prepared by pressureless infiltration technique, Composites, Part A, 2007, 38, 301-306.

$10 \mathrm{H}$. Wang, X. Zhou, B. Long and H. Liu, Compression behavior of $\mathrm{Al}_{2} \mathrm{O}_{3} \mathrm{k} / \mathrm{Al}$ composite materials fabricated by counter-gravity infiltration casting, Mater. Sci. Eng., A, 2013, 582, 316-320.

11 C. Ramshaw, The opportunities for exploiting centrifugal fields, Heat Recovery Syst. CHP, 1993, 13, 493-513.

12 E. C. G. German, V. D. S. John and A. K. Anton, A review on process intensification in HiGee distillation, J. Chem. Technol. Biotechnol., 2017, 92, 1136-1156.
13 G.-W. Chu, L. Sang, X.-K. Du, Y. Luo, H.-K. Zou and J.-F. Chen, Studies of $\mathrm{CO}_{2}$ absorption and effective interfacial area in a two-stage rotating packed bed with nickel foam packing, Chem. Eng. Process., 2015, 90, 34-40.

14 D. Zhang, P. Zhang, Z. Haikui, C. Guangwen, W. Wei, Z. Zhongwu, S. Lei and C. Jianfeng, Synthesis of petroleum sulfonate surfactant by different sulfonating agent with application of HIGEE technology, Chin. J. Chem. Eng., 2010, 18, 848-855.

15 C. Li, J. Gao, Z. Wang and Z. Guo, Separation of fine $\mathrm{Al}_{2} \mathrm{O}_{3}$ inclusion from liquid steel with super gravity, Metall. Mater. Trans. B, 2017, 48, 900-907.

16 Z. Wang, J. Gao, A. Shi, L. Meng and Z. Guo, Recovery of zinc from galvanizing dross by a method of super-gravity separation, J. Alloys Compd., 2018, 735, 1997-2006.

17 X. Huang, C. Liu, X. Lv, G. Liu and F. Li, Aluminum alloy pistons reinforced with $\mathrm{SiC}$ fabricated by centrifugal casting, J. Mater. Process. Technol., 2011, 211, 1540-1546.

$18 \mathrm{~J}$. Wannasin and M. Flemings, Fabrication of metal matrix composites by a high-pressure centrifugal infiltration process, J. Mater. Process. Technol., 2005, 169, 143-149.

19 K. Chang, J.-T. Gao, Z. Wang and Z.-C. Guo, Manufacturing 3-D open-cell aluminum foam via infiltration casting in a super-gravity field, J. Mater. Process. Technol., 2018, 252, 705-710.

20 A. Sánchez-Martínez, A. Cruz, M. González-Nava and M. Suárez, Main process parameters for manufacturing open-cell $\mathrm{Zn}-22 \mathrm{Al}-2 \mathrm{Cu}$ foams by the centrifugal infiltration route and mechanical properties, Mater. Des., 2016, 108, 494-500.

21 A. Paul and U. Ramamurty, Strain rate sensitivity of a closedcell aluminum foam, Mater. Sci. Eng., A, 2000, 281, 1-7.

22 J. Budke, Thermal etching of $\mathrm{NaCl}$ cleavage faces in vacuum, J. Am. Ceram. Soc., 1968, 51, 238.

23 J. Despois, A. Marmottant, L. Salvo and A. Mortensen, Influence of the infiltration pressure on the structure and properties of replicated aluminium foams, Mater. Sci. Eng., A, 2007, 462, 68-75.

24 C. Garcia-Cordovilla, E. Louis and J. Narciso, Pressure infiltration of packed ceramic particulates by liquid metals, Acta Mater., 1999, 47, 4461-4479.

25 M. Sánchez, J. Rams and A. Ureña, Fabrication of aluminium composites reinforced with carbon fibres by a centrifugal infiltration process, Composites, Part A, 2010, 41, 1605-1611.

26 R. Jamshidi-Alashti and G. Roudini, Producing replicated open-cell aluminum foams by a novel method of melt squeezing procedure, Mater. Lett., 2012, 76, 233-236.

27 R. Goodall, J.-F. Despois, A. Marmottant, L. Salvo and A. Mortensen, The effect of preform processing on replicated aluminium foam structure and mechanical properties, Scr. Mater., 2006, 54, 2069-2073.

28 R. Goodall, A. Marmottant, L. Salvo and A. Mortensen, Spherical pore replicated microcellular aluminium: processing and influence on properties, Mater. Sci. Eng., A, 2007, 465, 124-135. 